\title{
A group approach to genetic counselling of cardiomyopathy patients: satisfaction and psychological outcomes sufficient for further implementation
}

\author{
Ellen Otten ${ }^{\star, 1}$, Erwin Birnie ${ }^{1}$, Adelita V Ranchor ${ }^{2}, \mathrm{~J} \mathrm{Peter} \mathrm{van} \mathrm{Tintelen}^{1}$ and Irene M van Langen ${ }^{1}$
}

The introduction of next-generation sequencing in everyday clinical genetics practise is increasing the number of genetic disorders that can be confirmed at DNA-level, and consequently increases the possibilities for cascade screening. This leads to a greater need for genetic counselling, whereas the number of professionals available to provide this is limited. We therefore piloted group genetic counselling for symptomatic cardiomyopathy patients at regional hospitals, to assess whether this could be an acceptable alternative to individual counselling. We performed a cohort study with pre- and post-counselling patient measurements using questionnaires, supplemented with evaluations of the group counselling format by the professionals involved. Patients from eight regional hospitals in the northern part of the Netherlands were included. Questionnaires comprised patient characteristics, psychological measures (personal perceived control (PPC), state and trait anxiety inventory (STAI)), and satisfaction with counsellors, counselling content and design. In total, 82 patients (mean age 57.5 year) attended one of 13 group sessions. Median PPC and STAI scores showed significantly higher control and lower anxiety after the counselling. Patients reported they were satisfied with the counsellors, and almost $75 \%$ of patients were satisfied with the group counselling. Regional professionals were also, overall, satisfied with the group sessions. The genetics professionals were less satisfied, mainly because of their perceived large time investment and less-than-expected group interaction. Hence, a group approach to cardiogenetic counselling is feasible, accessible, and psychologically effective, and could be one possible approach to counselling the increasing patient numbers in cardiogenetics.

European Journal of Human Genetics (2015) 23, 1462-1467; doi:10.1038/ejhg.2015.10; published online 4 February 2015

\section{INTRODUCTION}

With the introduction of next-generation sequencing (NGS) in everyday clinical genetics practise, the number of genetic disorders that can be confirmed at DNA-level increases. This leads to a greater need for genetic counselling and testing of index patients and their close relatives, through cascade screening. However, the number of genetic professionals available cannot increase at the same rate, and other health professionals are generally insufficiently equipped for this task. This imbalance demands us to find new ways to effectively and efficiently accommodate to both the current and anticipated increase in requests for genetic counselling and testing, while maintaining the current levels of quality of care and patient satisfaction. This applies particularly to patients with relatively prevalent diseases, like cardiomyopathies, in which preventive options lead to a decrease in morbidity and mortality.

Hypertrophic cardiomyopathy (HCM) and dilated cardiomyopathy (DCM) are two common subtypes of cardiomyopathy. Prevalences are about 1:500 for HCM and the same or higher for DCM. ${ }^{1,2}$ Most HCM cases are familial, with (probable) causative genetic variants being found in up to $60 \%$ of familial and in $~ 30 \%$ of sporadic cases, using traditional sequencing techniques. ${ }^{3}$ In a Dutch cohort, the overall yield for familial and sporadic cases was $46 \%{ }^{4}$ About one-third of DCM cases are familial and a genetic cause can be found in $30-50 \%$ of these families. ${ }^{5,6}$ Familial cardiomyopathy mostly has an autosomal dominant mode of inheritance with reduced penetrance and variable expression, and preventive and treatment options are available. This makes cascade genetic testing of family members recommended practise. $^{3}$ Because NGS further increases the yield of causative genetic variants found in index patients, the number of family members being referred for genetic counselling and testing will also increase.

Group counselling is an attractive way of handling this increasing flow of patients to clinical genetics departments. It has been introduced in several medical disciplines, ${ }^{7}$ with the aim of providing information, patients sharing personal experiences, and increasing the efficiency of counselling for professionals. The general goals of genetic counselling are to increase patients' knowledge about their disease and its genetic aspects, and to ensure that patients can control their feelings about their situation/condition, resulting in the ability to make autonomous choices for themselves and their relatives. This is usually done in individual sessions, but could also be achieved group-wise as has been confirmed by experiences with breast cancer group counselling. ${ }^{8-11}$ These studies confirm that the aims and expected

${ }^{1}$ Department of Genetics, University Medical Center Groningen, University of Groningen, Groningen, the Netherlands; ${ }^{2}$ Department of Health Psychology, University Medical Center Groningen, University of Groningen, Groningen, the Netherlands

${ }^{*}$ Correspondence: E Otten, Department of Genetics, University Medical Center Groningen, University of Groningen, hanzeplein 1, P O Box 30001, Groningen 9700RB, the Netherlands. Tel: +31 50361 7229; Fax: +31 50361 7231; E-mail: e.otten@umcg.nl

Received 30 September 2014; revised 22 December 2014; accepted 7 January 2015; published online 4 February 2015 
advantages of group counselling can be reached. ${ }^{10}$ Patient satisfaction and the psychological outcomes of group genetic counselling are also generally positive. ${ }^{8-10}$ This oncogenetic group counselling mainly includes small heterogeneous patient groups that include both symptomatic and risk carriers. As far as we are aware, group counselling has not been piloted in cardiogenetics, despite being a rapidly growing segment of referrals.

We therefore piloted group genetic counselling for symptomatic cardiomyopathy patients as part of an intervention to increase access to genetic counselling and testing for patients and cardiologists at small regional hospitals in our service area. Our aim was to deliver adequate quality of care through group counselling. First, we wanted to determine whether the psychological benefits of group counselling were adequate, and at least comparable to the benefits of individual counselling. Second, we investigated both patients' and medical professionals' satisfaction with this type of counselling.

\section{METHODS}

\section{Study design and patients}

This was a cohort study with pre- and post-counselling measurements, conducted in eight small regional hospitals (mean 326 beds; range 197-642) in the northern part of the Netherlands (total area about $8304 \mathrm{~km}^{2}$ with about 1.7 million inhabitants). HCM and DCM index patients who had not attended genetic counselling and testing before, or those who felt the need for a second counselling, were eligible to participate. Thirteen group sessions took place between March 2011 and November 2012.

\section{Study process}

In the Netherlands, genetic counselling and testing for cardiomyopathies are performed by clinical geneticists and genetic counsellors employed at eight university medical centers (UMCs), in collaboration with psychosocial workers and dedicated cardiologists. This care is covered by compulsory, Dutch health insurance general policies. Cardiologists in nine regional hospitals within the service area of our university hospital were informed of the possibility of organising group counselling sessions in their hospital, and the formal referral criteria for genetic counselling and testing for symptomatic HCM and DCM patients were brought to their attention. ${ }^{12,13}$ Eight out of nine hospitals decided to participate.

Participating cardiologists and heart failure nurses selected eligible patients and informed them about the possibility of group genetic counselling. Interested patients received a standardized information letter about the possible genetic character of their cardiomyopathy and about the option for group genetic counselling and testing. Individual genetic counselling in the university hospital or at one of our three regular regional outpatient clinics was offered as an alternative. An application form, information-leaflet on group counselling and a stamped return-envelope were added. Patients who applied received an invitation. The preparations for group counselling were similar to those for standard individual care.

The counselling sessions were held in the afternoon or evening, in meeting rooms in the participating hospitals. All patients could bring one relative/ partner. At the beginning of the session, each participant was asked to sign a combined 'confidence declaration' and informed consent form to emphasise the confidential nature of the setting. Sessions lasted $\sim 2 \mathrm{~h}$, including completing the research questionnaires (taking about $30 \mathrm{~min}$ ) and a break. The legal department of our hospital approved the patient information material we compiled and the group counselling format regarding informed consent and privacy issues. Approval by a medical ethics committee was considered unnecessary because this care modality is also used in regular care.

The counselling team consisted of four professionals: (1) a clinical geneticistin-training provided the regular cardiogenetic and pre-test information, including a PowerPoint presentation, to patients and answered genetic questions, (2) a social worker was group leader and focused on group interactions and psychosocial issues of patients, (3) a local cardiologist and/or heart failure nurse was present to answer specific cardiological questions, and
(4) a clinical geneticist was present to supervise and assist with the short individual discussions after the group session had ended. These took about 5-10 min per patient and comprised recording additional personal and family history and discussion of personal considerations in DNA-testing. Immediately after the sessions, blood withdrawal for diagnostic DNA-testing was offered to all patients and performed in those who consented.

Group sessions differed from our standard individual counselling in three respects: (1) groups of unrelated patients were counselled together instead of individually, (2) counselling was done by a team instead of a single counsellor assisted by a social worker or cardiologist when necessary, and (3) counselling was given in the regional hospitals instead of at our department or regular outpatient clinics.

\section{Patient measurements}

The questionnaires before and immediately after the group counselling session requested the following information.

Patient characteristics. Patient's age, sex, number of offspring, educational level, diagnosis, and age at diagnosis.

Referral for genetic counselling. Patients were asked (1) who initiated their referral for genetic counselling and (2) if they had previously discussed referral for genetic counselling with their cardiologist.

Patient's questions. Before the group session began, patients were asked to indicate which questions they wanted to have answered during the session and if they had any additional questions that they preferred not to have discussed in the group. Afterwards, patients were asked which of their questions had been answered and which not, and if they had heard any information during the session that they would have preferred not to know.

We included five measures in the questionnaires to assess the experienced quality and the psychological outcomes of the counselling sessions in our patients:

Personal perceived control. The validated nine-item PPC (Dutch version) was used to measure patient's perceived control before and after genetic counselling $(\alpha=0.79-0.81){ }^{14}$ The response mode is a $0-2$ Likert scale. Higher scores indicate higher levels of perceived control.

State and trait anxiety inventory. The Dutch six-item short version of the STAI was used to measure patient's experienced anxiety before and after genetic counselling. The response mode is a 1-4 Likert scale, with higher scores indicating higher levels of anxiety. The convergent validity of the Dutch version of the STAI-6 with the full STAI showed a correlation of $0.95 \cdot{ }^{15,16}$

Clinical genetics satisfaction indicator (CGS). This seven-item questionnaire was adopted by the Clinical Genetics Association in the Netherlands to measure patient satisfaction with genetic counselling. The response mode is a 1-5 Likert scale. Higher scores indicate higher satisfaction. The English version shows excellent internal consistency in a clinical genetics setting $(\alpha=0.91) .{ }^{17}$ Internal consistency in the present study was 0.80 .

Patient preferences. Before the group counselling session, patients were asked to indicate their preference for the three main characteristics of group and individual counselling: (1) counselling in their local hospital versus counselling in the university hospital, (2) counselling by a team (geneticist, social worker and cardiologist) or by a single genetic counsellor, and (3) counselling in a group versus individually. They were also asked to indicate their overall preference for either group or individual counselling based on the three aspects jointly. The five-point response mode ranged from 'definite preference for group counselling' to 'no preference' to 'definite preference for individual counselling'.

Evaluation of content and design. Content was defined as both the appreciation of individual parts of the counselling and of the information given. Design concerned: (1) the appreciation of the entire process from referral to counselling, and (2) the composition of the group session itself and the presence of fellow patients. These were evaluated by means of a 20 -item ad hoc questionnaire, using a 0-2 Likert scale (totally agree/partly agree/disagree) as 
response mode. Higher scores indicated higher satisfaction with content and/or design of the group session. 'Being satisfied' was defined as 'sum-scores' $\geq 80 / 100$.

\section{Professionals' opinions}

At the end of the pilot period, all the professionals involved from the regional hospitals were sent an online questionnaire to evaluate the group sessions in terms of (1) their positive and negative experiences with the group sessions, (2) any feedback from patients, (3) suggestions for improvement, (4) willingness to organise further group sessions, and (5) willingness to refer patients to our university hospital for group counselling. We also asked them to rate their overall satisfaction with the group counselling session $(1=$ very unsatisfied to $10=$ very satisfied). The social worker, clinical geneticist, and clinical geneticistin-training evaluated the group counselling approach informally after each session, and more extensively at the end of the pilot period. Evaluation included feasibility and course of the sessions, interaction during the sessions, and their additional positive and negative experiences.

\section{Analysis}

The descriptive statistics used in this study were mean (SD) for variables with normal distributions, median (interquartile range) for variables with skewed distributions, and $n(\%)$ for nominal and ordinal variables. PPC, STAI, and GCS outcomes were analysed for patients who completed at least two-third of these questionnaires. Mean item scores per patient were calculated. Changes of PPC and STAI scores within patients were tested using the Wilcoxon rank-sum test for skewed variables and the paired Student's $t$-test for changes with normal distributions. We used the same tests to compare differences in PPC, STAI and CGS outcomes between small and large counselling groups $(<7$ versus $\geq 7$ patients). Estimated effect sizes were added. Data were analysed using SPSS statistics v20 (IBM Corporation, New York, NY, USA).

\section{RESULTS}

\section{Study process and sample}

During the study, 121 of the selected/approached patients were invited for a group counselling session (maximum $\sim 80 \%$; the exact number of patients selected/approached is unknown, as this was not fully reported by all cardiologists). Of those invited, 82 patients $(68 \%)$ attended one of the sessions, with 61 accompanying relatives/partners (Figure 1). Reasons for the 30 patients not attending their scheduled session ranged from not being interested in genetic counselling and testing on second thought $(n=4 ; 13 \%)$ to more practical reasons ( $n=17 ; 57 \%$; eg, sickness, having no transport, having other obligations at the time of the session), or simply not showing up for unknown reasons $(n=9 ; 30 \%)$. Median age of patients not showing up was 59.5 years (range 30-78), of whom $40 \%$ were male and $73 \%$ were referred because of DCM. The median age and proportion of DCM of these patients were comparable to the joining patients, whereas the no show-group did contain more females than the patients attending a group session. Median size of the group sessions was six patients (range 3-13 patients), and nine participants including accompanying relatives (range 5-27 persons). Four patients eventually attended an individual session at the university hospital instead of a group session; all because they were unable to be present at the scheduled group session and chose to attend the UMC for an individual session at short-term rather than waiting for a next group session at their own hospital. Patient characteristics are shown in Table 1. In total, 75 of 82 patients $(91 \%)$ completed both sets of questionnaires before and after the counselling session, whereas 81 of 82 patients completed at least one set. At the end of the pilot period, four cardiologists and two heart failure nurses from five of eight cooperating hospitals completed the professionals' evaluations.

\section{Patient measurements}

Referral for genetic counselling. Thirteen patients (16\%) reported having discussed genetic counselling with their doctor previously, but they did not attend/were not referred for genetic counselling until group genetic counselling was offered in their regional hospital. Three patients had been previously counselled individually at our university hospital and had DNA-testing initiated, but nevertheless wished to attend additional group genetic counselling.

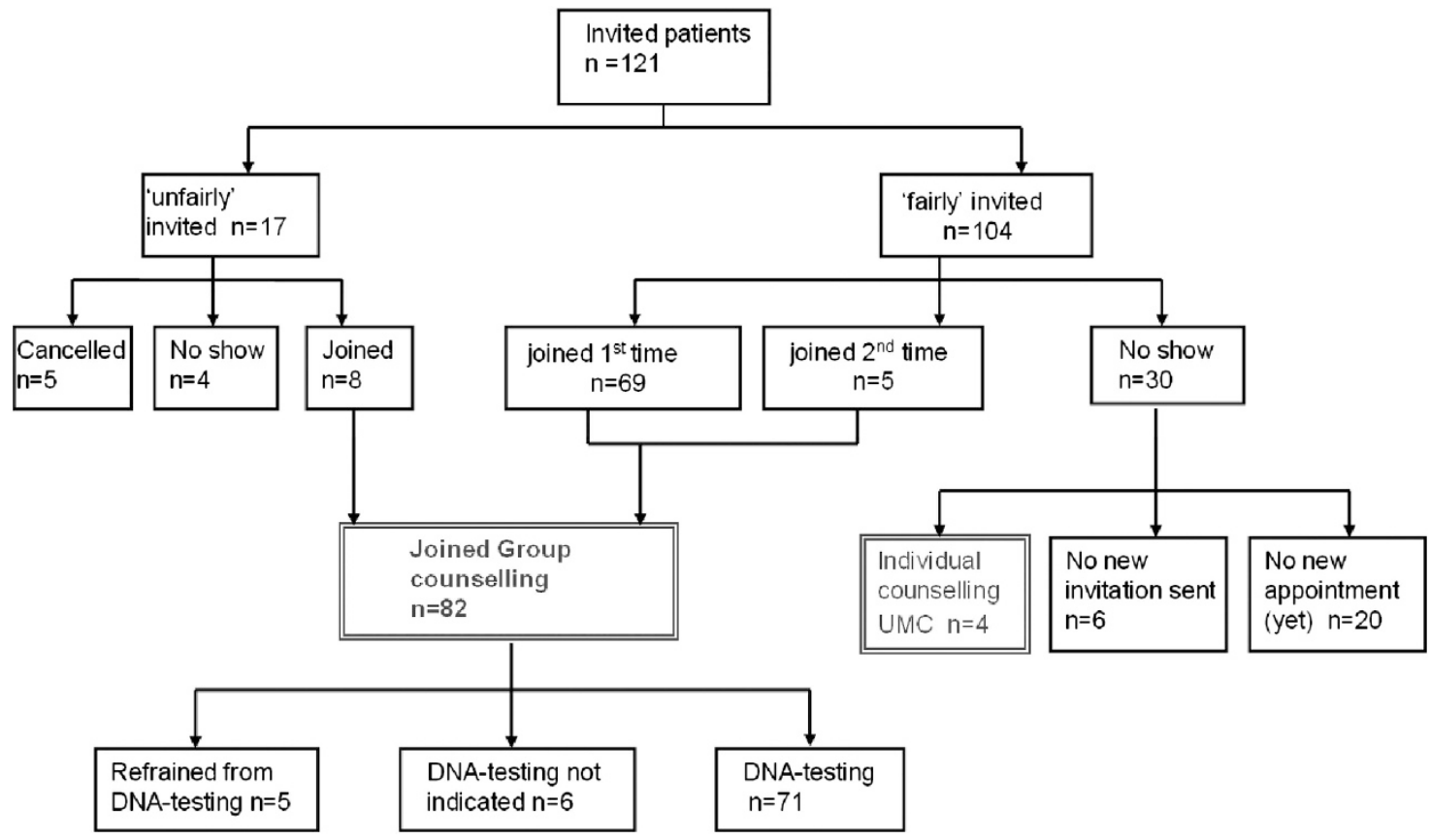

Figure 1 Overview of patients invited for a group counselling session. Legend: 'unfairly'= patients suspected of having a heritable cardiac disease, but not fulfilling the formal criteria for joining a group counselling session; 'fairly' = patients fulfilling the formal criteria for joining a group counselling session. 
Table 1 Characteristics of all patients attending group counselling sessions

\begin{tabular}{lr}
\hline Characteristic & \\
\hline Age (yr); median (range) & $57.5(20-79)$ \\
Sex; number male/female (\%) & $45 / 37(55 / 45 \%)$ \\
Patients with offspring (\% yes) & $71(89 \%)$ \\
& \\
Educational level & \\
$\quad$ Number of patients (\% of total) & \\
$\quad$ Low & $11(14 \%)$ \\
Intermediate & $58(73 \%)$ \\
$\quad$ High & $11(14 \%)$ \\
& \\
Cardiac disease & \\
Number of patients (\% of total) & \\
DCM & $52(63 \%)$ \\
HCM & $24(29 \%)$ \\
Other & $6(7 \%)$ \\
Time since diagnosis (yr); median (range) & $2.0(0-25)$ \\
\hline
\end{tabular}

Abbreviations: $\mathrm{DCM}=$ dilated cardiomyopathy; $\mathrm{HCM}=$ hypertrophic cardiomyopathy; $\mathrm{yr}=$ year . $n=2$ unknown.

Patient questions. Forty-two patients (51\%) reported at least one question they wished to have answered. Most questions addressed the consequences of confirming heritability for the patient and his/her close family members ( $n=22$ questions; $35 \%$ of questions) and cardiologic signs, symptoms and complaints $(n=17 ; 27 \%$ of questions). At the start of the session, one patient reported one topic, her personal situation, which she did not want to discuss during the group session. Afterwards, five patients reported having received general answers to their questions, but no specific answers for their personal situation. Only one patient reported having heard information he/she would have preferred not to know; this concerned the possibility/risk of sudden cardiac death in cardiomyopathy.

Psychological and satisfaction outcomes. Median change scores of the PPC were significantly higher (increased control) and those of the STAI were significantly lower (less anxiety) after counselling for all groups in relation to before the session (related samples Wilcoxon test, $P<0.001$ for both; Table 2), with effect sizes of 1.00 and 0.49 , respectively (Table 2 ). Patients also reported they were satisfied with the counsellors (Table 2); $56.5 \%$ of patients gave the maximum CGS questionnaire score of 5.0. PPC and STAI change scores and CGS scores did not differ significantly between small and large counselling groups (Independent Mann-Whitney $U$-test, $P=0.74, P=0.94$, and $P=0.31$, respectively; Table 2). The majority of patients indicated that they would join a group counselling session again if genetic counselling would be necessary $(87.7 \%)$, and would recommend it to family members $(81.4 \%)$.

Patient preferences. When comparing each main characteristic of our group counselling approach to its corresponding aspect in our individual counselling practise separately, almost half of the patients (48.5\%) preferred their local hospital over the university hospital, whereas $40 \%$ did not have any preference.

Regarding the involved professionals in each type of counselling, $52 \%$ did not have any preference for counselling by the multidisciplinary team or for counselling by just a single counsellor.

When taking all main characteristics of both counselling types into account, one-fourth of our patients preferred group counselling overall, whereas $30 \%$ preferred individual counselling overall.
Table 2 Psychological outcomes of patients attending group counselling sessions

\begin{tabular}{|c|c|c|c|c|}
\hline \multirow{4}{*}{$\begin{array}{l}\text { Outcome } \\
\text { measure }\end{array}$} & \multirow{4}{*}{$\begin{array}{l}\text { Time } \\
\text { point }\end{array}$} & \multicolumn{2}{|r|}{ Small groups } & \multirow{4}{*}{$\begin{array}{c}\text { Large groups } \\
(\geq 7 \text { patients) Median } \\
\text { score }(I Q R)\end{array}$} \\
\hline & & All groups & (<7patients) & \\
\hline & & Median score & Median score & \\
\hline & & (IQR) & $(I Q R)$ & \\
\hline \multirow[t]{4}{*}{$P P C$} & $\mathrm{n}$ & 64 & 34 & 30 \\
\hline & Before & $0.89(0.44)$ & $0.89(0.44)$ & $0.78(0.50)$ \\
\hline & After & $1.24(0.56)$ & $1.39(0.47)$ & $1.17(0.44)$ \\
\hline & $\Delta$ & $0.33(0.44)$ & $0.41(0.42)^{a}$ & $0.30(0.47)^{a}$ \\
\hline \multirow[t]{4}{*}{ STAI } & $n$ & 67 & 36 & 31 \\
\hline & Before & $1.67(0.67)$ & $1.83(0.67)$ & $1.67(0.70)$ \\
\hline & After & $1.67(0.83)$ & $1.67(0.63)$ & 1.50 (1.17) \\
\hline & $\Delta$ & $0.17(0.33)$ & $0.08(0.50)$ & $0.17(0.33)$ \\
\hline \multirow[t]{2}{*}{ CGS } & $\mathrm{n}$ & 69 & 37 & 32 \\
\hline & After & $5.0(0.50)$ & $5.0(0.29)$ & $5.0(0.71)$ \\
\hline
\end{tabular}

Abbreviations: After $=$ immediately after session; Before $=$ at start of session; $C G S=$ clinical genetics satisfaction indicator; $I Q R=$ interquartile range; $S T A I=$ state and trait anxiety inventory; $\Delta=$ change between before and after; $n=$ number of patients; PPC = personal perceived control $\Delta=$ change between before and after; $n=$ number of patients; $P P C=$ personal perceived control
questionnaire. Significant changes $(P<0.05)$ are displayed in bold, test used = Wilcoxon test, except where indicated.

astudent's $t$-test.

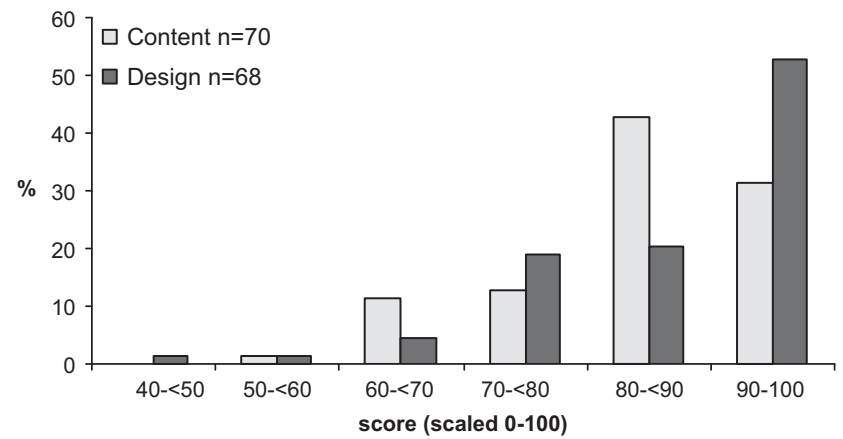

Figure 2 Patient satisfaction with content and design of group counselling sessions.

The remaining patients (45.3\%) did not have a clear overall preference for one of both counselling types.

Evaluation of content and design. Almost three-quarters of our patients reported being satisfied with the content (74.3\%) and design (73.4\%) of the group counselling session (Figure 2). One patient reported the design of the group session process being overall insufficient (score 41 of 100). The items with mean lowest success scores in all patients (range 1-5) were 'usefulness of discussing DNAtesting with fellow patients' (mean score 1.26) and 'experiencing support from fellow patients' (mean score 1.11).

Professionals' evaluations. Regional professionals reported they were satisfied overall with the course of the group counselling at their hospital (mean score 7.8). No negative reactions from their patients were reported. Suggestions for improvement were to be clearer about the session aims (emphasis on genetic information, not on cardiac information), to shorten its duration (shorter introduction), and to consider optimal group size (maximum 10 patients) and time of day (symptomatic patients may be too tired by the end of the day). 
All professionals indicated they were willing to organise group counselling sessions for index patients in the future. One of six professionals was not willing to refer patients to the university hospital for group counselling because she thought the regional group concept is more suitable for increasing access to genetic counselling.

The genetics professionals involved were less enthusiastic about the group counselling format than their regional cardiologic colleagues: one positive aspect mentioned was that more, and possibly different, patients were reached and informed by this new approach. Perceived disadvantages were that group counselling in eight hospitals was less efficient than counselling at the university hospital (mainly due to travelling time), that the practical organisation of the sessions was difficult and time-consuming, and that it was sometimes difficult to stimulate the group interaction.

\section{DISCUSSION}

We evaluated the quality and satisfaction of group genetic counselling for symptomatic cardiomyopathy patients. The outcomes show that the group counselling participants experienced good quality of care, that group counselling was not harmful, and that the psychological aims were met. Moreover, the professionals involved from the regional hospitals were overall positive about this way of providing genetic counselling. Finally, our group counselling approach seemed feasible, acceptable, and satisfied a need in the view of the number of patients that attended group counselling.

As far as we know, we are the first to have piloted and evaluated group counselling in cardiogenetic patients. We included a substantial number of patients to support the reliability of our results. The participants were heterogeneous in terms of gender, age, and educational level, which suggests that, overall, group counselling is acceptable to a wide range of patients. Finally, our pilot study closely mimicked daily practise, that is, the barriers we faced probably also occur in daily practise. This makes our group approach relatively easy being integrated in existing clinical workflow (Figure 3).

Reference PPC and STAI data for cardiomyopathy patients are unavailable. The psychological outcomes of our patients showed suboptimal baseline as well as post-counselling levels of PPC scores, and better STAI scores than reports on individually counselled oncogenetic patients in the Netherlands. However, the improvements in scores between pre- and post-counselling (within patients) were similar. ${ }^{9,14,8-20}$ Our PPC results in cardiogenetics are also comparable to group counselling in oncogenetics, ${ }^{9}$ and several reports from the United Kingdom about STAI outcomes in oncogenetics show similar improvements in scores as in our patients. ${ }^{21-23}$ The psychological outcomes of our patients may have been influenced by group size, group dynamics, and/or the management of questions which they did or did not want to be addressed during the group session. But this all seems unlikely as the psychological outcomes of both the smaller and larger groups in our study were about equal (Table 2). Only one patient indicated a topic she preferred not to be discussed and only one patient reported having heard some information he would have preferred not to know. This leads us to conclude that our patients overall accept group counselling psychologically and are not harmed by it.

In addition to the positive outcomes of group counselling reported by the patients and the regional professionals, our approach had some indirect effects. By bringing genetic counselling closer to our patients and the regional professionals, we lowered the threshold for attending genetic counselling, and we provided education and created awareness in the regional professionals, which hopefully will have an ongoing beneficial effect on their referral rates.

Unlike the patients and the regional professionals, the genetics professionals experienced several drawbacks with group counselling, particularly a greater time investment and less interaction among patients than expected. Additional research is needed, with regard to time investment, to determine the most appropriate format of the group counselling sessions for both the patients and professionals involved. Sessions could be adapted regarding design, duration, and/or location, to meet the requirements of both patients and professionals. Regarding patient interactions, our expectations were perhaps too much influenced by the literature on repeated group sessions, for example, in diabetic care. ${ }^{7,24}$ A minimum level of group bonding is needed before patients will share thoughts and experiences: this is more likely to evolve over the course of multiple sessions. Moreover, the limited group interaction might also be partly due to the fact that most of these symptomatic patients had already decided for genetic testing before attending the group session, making group interaction less important for them. However, the patient outcomes for the counselling sessions did not suffer from the limited interaction, as shown by the PPC and STAI results. The degree of interaction might be different in group counselling of relatives considering predictive testing, but this has yet to be investigated.

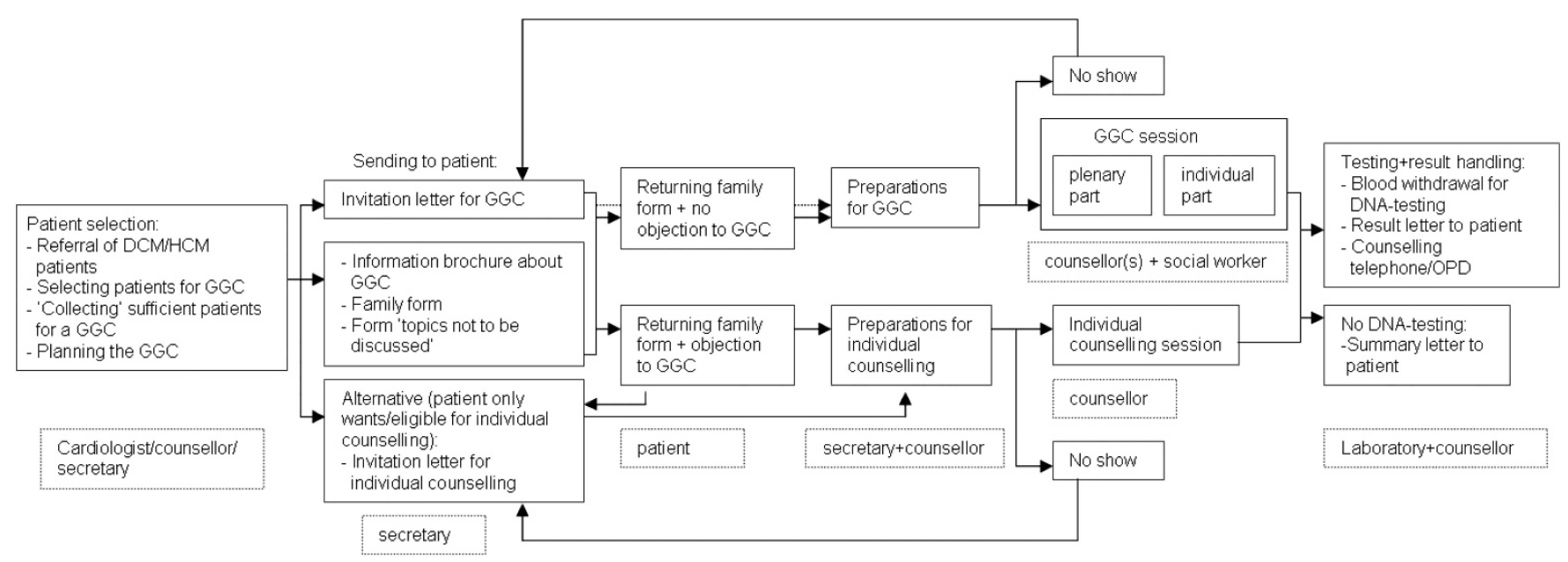

Figure 3 Flow chart - integration of the group genetic counselling process into routine care. 
One study limitation is that we cannot be certain that our results are unbiased, owing to the absence of a matched control group that received individual counselling. Moreover, PPC and STAI changes were evaluated directly after the counselling sessions and we did not evaluate the persistence of these benefits in the longer term. Our results may also have been subject to participation bias, as the patients chose to join group counselling rather than refusing this type of counselling outright.

In conclusion, in the near future results of NGS diagnostics will become available for large numbers of HCM and DCM patients, and for patients with other (common) genetic diseases. Because of this anticipated increase, together with growing awareness of these growing possibilities, we should be prepared for an increased demand for genetic counselling and testing of patients and their family members. Fulfilling these needs is important in the light of achieving health gains. Our results support our hypothesis that group genetic counselling in cardiogenetics is a feasible, accessible, and psychologically effective way of counselling large numbers of symptomatic cardiomyopathy patients. Further research is needed to determine which type of counselling will be most suitable in view of increasing patient numbers, thereby satisfying both patients' and professionals' needs, maintaining at least current levels of quality and of access to clinical genetic care, and being cost-effective.

\section{CONFLICT OF INTEREST}

The authors declare no conflict of interest.

\section{ACKNOWLEDGEMENTS}

We are grateful to Jo Jacobs, psychosocial worker, for her participation and cooperation in this pilot project. We thank the participants in the group sessions and those who completed questionnaires, and the regional cardiologists and heart failure nurses for their cooperation. We also thank Jackie Senior and Kate McIntyre for editing our manuscript.

1 Maron BJ, Maron MS, Semsarian C: Genetics of hypertrophic cardiomyopathy after 20 years: clinical perspectives. J Am Coll Cardiol 2012; 60: 705-715.

2 Mestroni L, Taylor MR: Genetics and genetic testing of dilated cardiomyopathy: a new perspective. Discov Med 2013; 15: 43-49.

3 Ackerman MJ, Priori SG, Willems S et al: HRS/EHRA expert consensus statement on the state of genetic testing for the channelopathies and cardiomyopathies: this document was developed as a partnership between the Heart Rhythm Society (HRS) and the European Heart Rhythm Association (EHRA). Europace 2011; 13: 1077-1109.

4 Hofman N, Tan HL, Alders M et al: Yield of molecular and clinical testing for arrhythmia syndromes. Report of 15 years' experience. Circulation 2013; 128: 1513-1521.

5 van Spaendonck-Zwarts KY, van Rijsingen IA, van den Berg MP et al: Genetic analysis in 418 index patients with idiopathic dilated cardiomyopathy: overview of 10 years' experience. Eur J Heart Fail 2013; 15: 628-636.

6 Pugh TJ, Kelly MA, Gowrisankar S et al: The landscape of genetic variation in dilated cardiomyopathy as surveyed by clinical DNA sequencing. Genet Med 2014; 16: $601-608$
7 Jaber R, Braksmajer A, Trilling JS: Group visits: a qualitative review of current research. J Am Board Fam Med 2006; 19: 276-290.

8 Ridge Y, Panabaker K, McCullum M, Portigal-Todd C, Scott J, McGillivray B: Evaluation of group genetic counseling for hereditary breast and ovarian cancer. J Genet Couns 2009; 18: 87-100.

9 Rothwell E, Kohlmann W, Jasperson K, Gammon A, Wong B, Kinney A: Patient outcomes associated with group and individual genetic counseling formats. Fam Cancer 2012; 11: 97-106.

10 Calzone KA, Prindiville SA, Jourkiv 0 et al: Randomized comparison of group versus individual genetic education and counseling for familial breast and/or ovarian cancer. J Clin Oncol 2005; 23: 3455-3464.

11 Visser A, Prins JB, Hoogerbrugge N, van Laarhoven HW: Group medical visits in the follow-up of women with a BRCA mutation: design of a randomized controlled trial. BMC Womens Health 2011; 11: 39.

12 Mestroni L, Maisch B, McKenna WJ et al: Guidelines for the study of familial dilated cardiomyopathies. Collaborative Research Group of the European Human and Capital Mobility Project on Familial Dilated Cardiomyopathy. Eur Heart J 1999; 20: 93-102.

13 ICIN working group on Hereditary Heart Diseases: Genetic diagnostics and genetic counselling in Hypertrophic Cardiomyopathy (HCM). Neth Heart J 2010; 18: 144-159.

14 Smets EM, Pieterse AH, Aalfs CM, Ausems MG, van Dulmen AM: The perceived personal control (PPC) questionnaire as an outcome of genetic counseling: reliability and validity of the instrument. Am J Med Genet A 2006; 140: 843-850.

15 van der Bij AK, de Weerd S, Cikot RJ, Steegers EA, Braspenning JC: Validation of the Dutch short form of the state scale of the Spielberger State-Trait Anxiety Inventory: considerations for usage in screening outcomes. Community Genet 2003; 6: 84-87.

16 Spielberger CD: Manual for the State-Trait Anxiety Inventory STAI (Form Y). Palo Alto, CA, USA: Consulting Psychologists Press, 1983.

17 Zellerino B, Milligan SA, Brooks R, Freedenberg DL, Collingridge DS, Williams MS: Development, testing, and validation of a patient satisfaction questionnaire for use in the clinical genetics setting. Am J Med Genet C Semin Med Genet 2009; 151C: 191-199.

18 Pieterse AH, van Dulmen AM, Beemer FA, Bensing JM, Ausems MG: Cancer genetic counseling: communication and counselees' post-visit satisfaction, cognitions, anxiety, and needs fulfillment. J Genet Couns 2007; 16: 85-96.

19 Zilliacus EM, Meiser B, Lobb EA et al: Are videoconferenced consultations as effective as face-to-face consultations for hereditary breast and ovarian cancer genetic counseling? Genet Med 2011; 13: 933-941.

20 Braithwaite D, Emery J, Walter F, Prevost AT, Sutton S: Psychological impact of genetic counseling for familial cancer: a systematic review and meta-analysis. Fam Cancer 2006; 5: 61-75.

21 Brain K, Gray J, Norman P et al: Randomized trial of a specialist genetic assessment service for familial breast cancer. J Natl Cancer Inst 2000; 92: 1345-1351.

22 Bish A, Sutton S, Jacobs C, Levene S, Ramirez A, Hodgson S: Changes in psychological distress after cancer genetic counselling: a comparison of affected and unaffected women. Br J Cancer 2002; 86: 43-50.

23 Cull A, Anderson ED, Campbell S, Mackay J, Smyth E, Steel M: The impact of genetic counselling about breast cancer risk on women's risk perceptions and levels of distress. Br J Cancer 1999; 79: 501-508.

24 Raballo M, Trevisan M, Trinetta AF et al: A study of patients' perceptions of diabetes care delivery and diabetes: propositional analysis in people with type 1 and 2 diabetes managed by group or usual care. Diabetes Care 2012; 35: 242-247.

(1) (5) (2) This work is licensed under a Creative Commons Attribution-NonCommercial-ShareAlike 3.0 Unported License. The images or other third party material in this article are
included in the article's Creative Commons license, unless indicated otherwise in the credit line; if the material is not included under the Creative Commons license, users will need to obtain permission from the license holder to reproduce the material. To view a copy of this license, visit http://creativecommons.org/licenses/by-nc-sa/3.0/ 